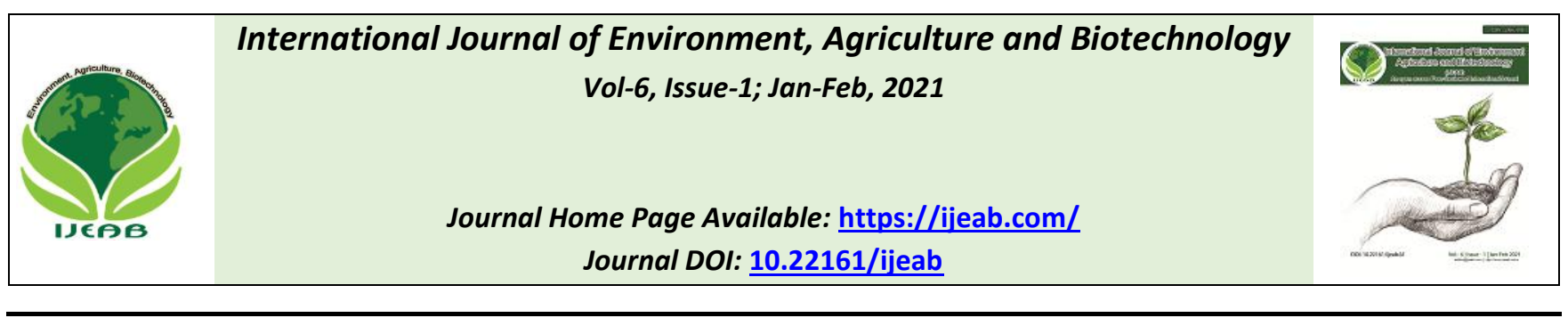

\title{
Yeast Extract: A Potential Substitute of Salt in Baked Crackers
}

\author{
Loubna Abou Ghoush and Sami El Khatib*
}

Department of Food Science and Technology. School of Arts and Sciences Lebanese International University. Al Khiara, West Bekaa, Lebanon

*Corresponding Author

Received: 01 Dec 2020; Received in revised form: 12 Jan 2021; Accepted: 20 Jan 2021; Available online: 29 Jan 2021

(C)2021 The Author(s). Published by Infogain Publication. This is an open access article under the CC BY license

(https://creativecommons.org/licenses/by/4.0/).

\begin{abstract}
Yeast extract (YE) is a natural product that can be easily produced and has the advantage to be edible and to have a salty taste. It can be used as a food additive to give Umami taste, to intensify flavor and to give saltiness. YE can be produced naturally by autolysis, (a self lysis of the yeast cells) when incubated for four days at $45^{\circ} \mathrm{C}$ for the yeast cells to release their cytoplasmic content. YE is low in calories and fat, high in proteins, nucleotides and glutamic acid. It is used by food companies and restaurants as an aromatic ingredient in various applications such as soups, sauces, seasonings, snacks, ready meals and meats. The main objective of this study is to reduce the salt concentration and replace it by the edible YE in the salty crackers. In our experiment, salt concentration was reduced by 25\%, 50\%, $75 \%$ and $100 \%$ and replaced with YE. Physico-chemical properties of the product were investigated and sensory evaluation was performed by a set of 25 panelists. Triangle test and consumers' acceptance test were performed by comparing experimental sample against the control containing $100 \%$ salt. Obtained results supported the hypothesis; the taste of samples with 50\% reduced salt showed no difference compared to those made using YE. The panelists gave the latter sample six points out of nine on hedonic scale. Our findings demonstrated that YE can be potentially used as a partial substitute of table salt.
\end{abstract}

Keywords - Yeast Extract, Salt, Monosodium Glutamate, Umami Taste, Crackers.

\section{INTRODUCTION}

For many years and from a sensory perspective, salt had many desirable properties. Salt is a cheap ingredient that improves the sensory properties of every food that humans consume. It enhances the positive sensory attributes of foods even the unpalatable ones and makes them taste better. However, salt causes hypertension (Liem et al., 2011), water retention (Sunil et al., 2013), increased heart pressure (Lehnhardt \& Kemper, 2011), edema and kidney failure (Trayes et al., 2013). Due to its availability in many processed foods, it is highly consumed. Several studies tried to decrease the salt concentration in food; the process was done gradually to be able to monitor the preservation of the salt effects on food (Henney, 2010).

Replacing salt with other substances represented a great interest for food producers. Yeast extract (YE) is one of the acceptable replacers of salt, as a natural product that can be produced easily (Taylor et al., 2018).

YE can be produced by different methods, mainly by enzymatic treatment, chemical treatment, sonication, plasmolysis or by thermolysis methods (Padmakumara, 2006). For the purpose of replacing salt with YE, this latter should be naturally produced in order to be edible and safe to consume. Autolysis is a fully natural method and gives an edible yeast extract, by only controlling two parameters, the temperature and the time of incubation (Padmakumara, 2006).

$\mathrm{YE}$ is a brown liquid that can be stored at $-20^{\circ} \mathrm{C}$ for later usage. When spray dried, it gives a brown powder that shows a saltier taste and better effect in replacing salt. In order to evaluate the effect of saltiness of YE, it should be tested on a salty product such as crackers. Consumers buy 
crackers as a salty snack, that is available in many flavors such as oregano and garlic, tomato and olives, basil and others (Mihalos, 2014).

Savory and salty snacks consumption has increased as a result of urbanization and modernization (Mihiranie et al., 2017). Crackers are popular as a healthy salty snack with a high potential to enhance their nutritional value by incorporating natural ingredients such as flavors(Mihiranie et al., 2017). People who cannot consume high amounts of salt seek alternative crackers that have the same salt taste but with less salt concentration.

The main objective of this study is to reduce the salt concentration of crackers and replace the reduced amount by YE. The commercially available crackers were used as a reference. All samples were prepared following the same recipe and under the same processing conditions. The sensory evaluation studies determined the acceptable level at which YE can replace salt.

In our study, the salt was decreased gradually and eventually replaced by dried YE. Different mixes were obtained by reducing salt concentration by $20 \%$, $50 \%$, $75 \%$ and $100 \%$, and replacing the reduced amount with dried YE. Two sensory analysis tests were performed (the triangle test and the consumer's acceptance test) in order to evaluate the effect of YE on giving the same saltiness of the original crackers.

The physiochemical characteristics of YE were determined. Salinity, $\mathrm{pH}$ value, nitrogen amount, fat content, moisture content, density, amount of calories, nucleotides content, glutamic acid concentration and moisture content were all studied in order to fully characterize the YE.

\section{MATERIALS AND METHODS}

\section{Preparation of Yeast Extract (YE)}

The yeast strain Saccharomyces cerevisiae known as baker's yeast and used in many baked products, was subject to autolysis and then to spray drying before usage. The liquid yeast extract was used for the physiochemical studies, and the dried yeast extract was used for baking.

\section{Autolysis of Yeasts}

Autolysis is a self-digestion process that encourages the breakdown of cells by their endogenous enzymes. It is a natural process that can take place under specific conditions of $\mathrm{pH}$, temperature or salt concentration (Padmakumara, 2006). For our purpose, autolysis has been initiated as described by Padmakumara. Dissolve $125 \mathrm{~g}$ of dry yeast Saccharomyces cerevisiae in 500ml distilled water as shown in the Figure 1. Incubate for four days at $45^{\circ} \mathrm{C}$ then collect the supernatant (Figure 2) and centrifuge it for 15 minutes at $4000 \mathrm{rpm}$.

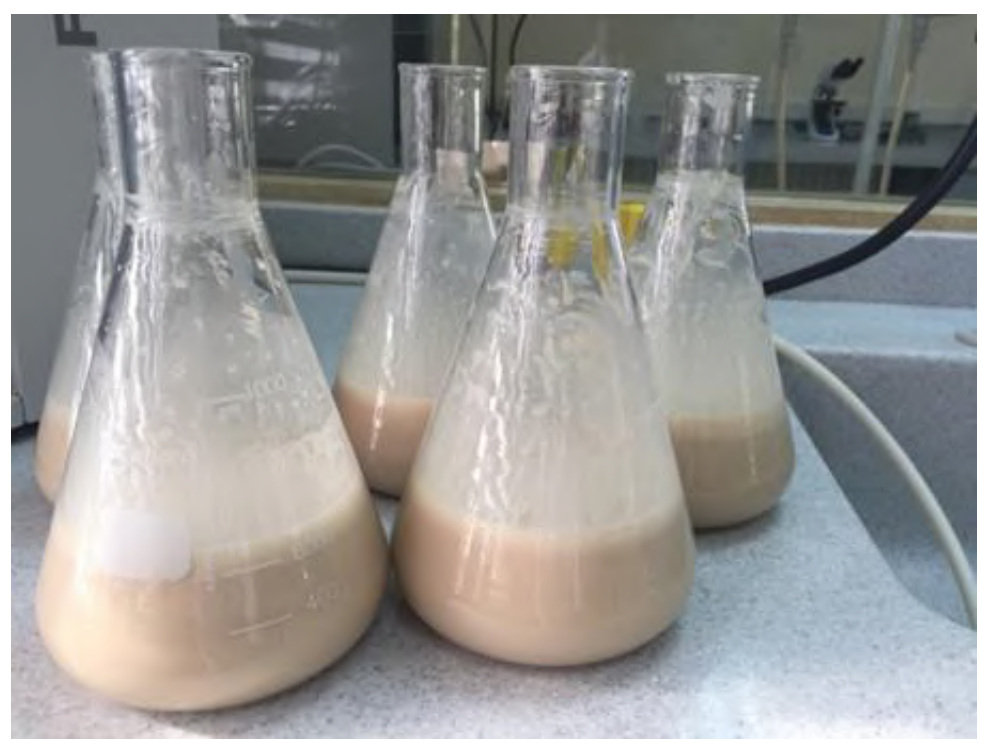

Fig.1: Dissolved yeast in distilled water prepared in our research lab

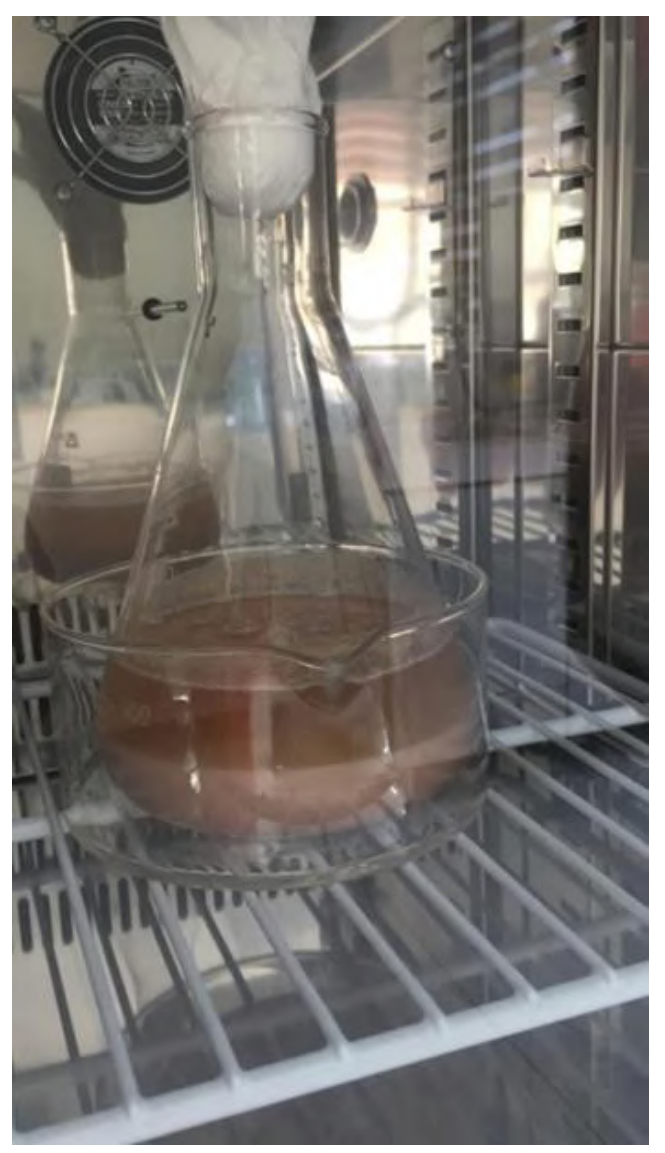

Fig.2: Yeast extract after 4 days of incubation, before separating the extract from the cell debris.

Spray Drying

Spray drying was used to produce a dry powder yeast extract from the liquid extract. The pre- prepared liquid 
yeast extract was used. The process was conducted as described by Tanguler and Erten(Tangüler \& Erten, 2009).

\section{Characterization of Yeast Extract}

\section{Salinity of Yeast Extract}

The electro conductivity meterfrom ORION 420APLUS meterwas used to measure the value of current that passes through the liquid yeast extract. Based on the flow of the current, the concentration of ions found in a solution can be evaluated and then used to assess the amount of salt present in different concentrations of liquid yeast extract. Results are recordedin microseimens (ms). The value obtained in ms is equal to milliohms; multiplied by 1000 to convert it tomicroomhs. To get the concentration in ppm, multiply the number by 0.64 , and then divide it by 1000 to get the concentration in $\mathrm{g} / \mathrm{L}$.Concentrate $80 \mathrm{ml}$ of liquid yeast extract by reducing water, using a water bath at $45^{\circ} \mathrm{C}$. Measure salinity at $80,60,40$ and $20 \mathrm{ml}$ and convert it from $\mathrm{ms}$ to $\mathrm{g} / \mathrm{L}$.

\section{pH of Yeast Extract}

The $\mathrm{pH}$ of the liquid yeast extract was measured using HannapH meter. Measure $30 \mathrm{ml}$ of the yeast extract in a graduated cylinder.Put the cylinders in water bath at $45^{\circ} \mathrm{C}$ to concentrate the yeast extract into different volumes and measure the $\mathrm{pH}$ at each volume. Record the results (Table 1)

Table 1: Measured pH values of different concentrations of liquid $Y E$.

\begin{tabular}{|c|c|c|c|c|}
\hline $\begin{array}{c}\text { Volume } \\
\text { ml }\end{array}$ & \multicolumn{3}{|c|}{ pH } & \multirow{2}{*}{ Average } \\
\cline { 1 - 3 } Replicates & $\mathbf{1}$ & $\mathbf{2}$ & $\mathbf{3}$ & \\
\hline $\begin{array}{c}30 \mathrm{ml} \\
(100 \%)\end{array}$ & 5.39 & 5.08 & 5.10 & $5.19 \pm 0.1$ \\
\hline $\begin{array}{c}24 \mathrm{ml} \\
(80 \%)\end{array}$ & 5.37 & 5.37 & 5.38 & $5.37 \pm 0.005$ \\
\hline $\begin{array}{c}18 \mathrm{ml} \\
(60 \%)\end{array}$ & 5.39 & 5.41 & 5.39 & $5.39 \pm 0.01$ \\
\hline $\begin{array}{c}12 \mathrm{ml} \\
(40 \%)\end{array}$ & 5.33 & 5.40 & 5.33 & $5.35 \pm 0.04$ \\
\hline $\begin{array}{c}6 \mathrm{ml} \\
(20 \%)\end{array}$ & 5.53 & 5.54 & 5.52 & $5.53 \pm 0.01$ \\
\hline
\end{tabular}

\section{Determination of Fat by Soxhlet}

Soxhlet is a process of continuous solid/liquid extraction. Powdered yeast extract previously prepared by spray drying, was placed in a thimble that allows the liquid to pass through. The Soxhlet apparatus FoodAlyt, model RS 40 was used to determine the fat content. The process was conducted as described in the FAO Manuals of Food Quality Control with some modifications. Weigh thimble + sample before extraction and record the values. Weigh the empty flasks where the solvent will be placed and record masses. Then measure $150 \mathrm{ml}$ petroleum ether and introduce it into the flasks.Place the thimbles and flasks in the Soxhlet extraction machine and run it for 4 hours. Turn off the machine and let it cool completely.Allow petroleum ether to evaporate from the flasks using a water bath from J.P SELECTA, Spain.Measure the mass of the thimble and sample and the mass of the empty flasks after extraction. Calculate the fat amount extracted by subtracting the weight of the thimble after extraction from its weight before extraction.

\section{Moisture Content}

Use the moisture analyzer RADWAG of model number MA 210.R that measures the amount of moisture present in a sample of dried YE used in crackers baking.

\section{Determination of Total Nitrogen Content by Kjenldahl}

The kjendahl method is used to determine the nitrogen content in organic and inorganic samples. This procedure has high precision and simple application. It consists of three major steps.

The first step is a digestion process by acid at high temperature with the help of catalyst to speed up the reaction. The higher the temperature, the faster the reaction. The digestion procedures were performed using FoodAlyt SBS800 Kjendahl Digestion Unit. The process was done as stated by the FAO Manual for Food Quality Control. In a $250 \mathrm{ml} \mathrm{Kjendhl} \mathrm{test} \mathrm{tube} \mathrm{add} 1 \mathrm{~g}$ of powdered yeast extract. Add some glass beads, $20 \mathrm{ml}$ of sulfuric acid and one Kjendahl tablet (catalyst). Prepare three replicates and one blank flask that contains all of the above except the sample. The temperature was set at $420^{\circ} \mathrm{C}$. Run the process for 4.5 hours or until the fumes disappear and the color of the sample turns transparent.

The second step is a process of distillation. After cooling the flasks of the Kjendahl, the FoodAlyt D4000 unit was used to perform the distillation with the following parameters: $50 \% \mathrm{NaOH}, 4 \%$ boric acid and Methyl redbromocresol as an indicator. Put each sample in the distillator for five minutes.

The third step is a process of titration. Direct titration takes place using $0.1 \mathrm{M} \mathrm{HCl}$, and the equivalence point is detected when color change occurs. The volumes of the used acid were recorded and the nitrogen percentage was calculated.

\section{Determination of calories by bomb calorimeter}

Bomb calorimeteris used to efficiently measure the heat released by combustion reactions. It is a sealed vessel that 
contains a smaller container called a "bomb". This machine CAL3K-U calorimeter was used to determine the calories found in the powdered yeast extract. The procedure was performed as described by Mack and Black as follows:Weigh $0.5 \mathrm{~g}$ of powdered yeast extract and put it in the sample cup. Add $0.5 \mathrm{~g}$ benzoic acid to the sample.Tie the cotton rod around the iron rod above the sample cup, and make sure that the cotton rod is in touch with the benzoic acid tablet.

Tightly close the bomb and put it in the filling station to fill it with oxygen. Then put the bomb in the calorimeter and run it.Repeat the procedure to ensure three replicates.

The values are obtained in Joules and then converted into Kcal by dividing the value by 4.184 .

\section{Determination of Nucleotides Concentration}

The determination of the concentration of the nucleotides 5'IMP (inosine 5'-monophosphate) and 5'GMP (guanosine 5'-monophosphate) has been carried out by spectrophotometric method. Pereira and his colleagues found that the best absorbance of IMP and GMP can be obtained at wavelength of $250 \mathrm{~nm}$ (Pereira et al., 2018). Therefore, the absorbance of IMP and GMP was measured at 250nm using Thermo Electron Corporation, model number Genesys 10-S in liquid yeast extract sample.

Two standard curves were plotted to calculate the exact amount of nucleotides found in our sample. Different concentrations of both nucleotides were prepared by dissolving different masses of both in distilled water, as shown in the table below:

Table 2: Masses of IMP and GMP used to prepare standard concentrations of both solutions.

\begin{tabular}{|c|c|c|}
\hline Concentration & Mass of 5'IMP & Mass of 5'GMP \\
\hline $0.2 \mathrm{M}$ & $0.85 \mathrm{~g}$ & $0.81 \mathrm{~g}$ \\
\hline $0.4 \mathrm{M}$ & $1.71 \mathrm{~g}$ & $1.62 \mathrm{~g}$ \\
\hline $0.6 \mathrm{M}$ & $2.56 \mathrm{~g}$ & $2.44 \mathrm{~g}$ \\
\hline $0.8 \mathrm{M}$ & $3.42 \mathrm{~g}$ & $3.25 \mathrm{~g}$ \\
\hline $1 \mathrm{M}$ & $4.28 \mathrm{~g}$ & $4.07 \mathrm{~g}$ \\
\hline
\end{tabular}

Then the absorbance of each concentration was measured at $250 \mathrm{~nm}$ and the standard curves were plotted. According to these curves, the concentrations of nucleotides in our sample were determined.

\section{Determination of Glucose Concentration}

The refractometry method gives the total amount of glucose found in a sample. A protocol described by
Laulloo and his colleagues (Laulloo \& Cadet, 2003) was applied to measure the glucose amount in the liquid yeast extract, with some modifications, as follows:Use Hanna model number HI 96801 refractometer, then prepare standard solutions of glucose of concentrations $0.1,0.3$, $0.5,0.7$ and $0.9 \mathrm{~g} / \mathrm{ml}$.Measure the refractive index of each concentration three times, and plot a standard curve of concentration of glucose versus refractive index percentage. After that, measure the refractive index of the liquid yeast extract three times and plot it on the curve and calculate the concentration of glucose found in it.

\section{Determination of Glutamic Acid Concentration}

Earlier, Shah and his colleagues reported that amino acid reacts with acetylacetone-formaldehyde reagent to generate a yellow color product (Shah, 1992). They evaluated the effect of different parameters on this reaction to obtain maximum color intensity. As they stated in their protocol, the amount of glutamic acid was determined by first preparingacetylacetone-formaldehyde reagent solution by adding $7.8 \mathrm{ml}$ acetylacetone to $15 \mathrm{ml}$ formaldehyde $36 \% \mathrm{w} / \mathrm{w}$. Then $100 \mathrm{mg}$ of powdered yeast extract was weighed and dissolved in $100 \mathrm{ml}$ distilled water in an Erlenmeyer flask and add to it $3 \mathrm{ml}$ of the reagent.Put the Erlenmeyer flask in a boiling water bath.After 20 minutes, remove the Erlenmeyer from the water bath and measure and record the absorbance using at $415 \mathrm{~nm}$.Prepare different concentrations of glutamic acid and repeat the same procedures to form a standard curve, measure the absorbance of the different concentrations at $450 \mathrm{~nm}$ and plot the standard curve.From the standard curve, calculate the amount of glutamic acid found in the powdered yeast extract.

\section{Baking of Crackers}

Recipe of Crackers

- Ingredients:

1 cup all-purpose flour, 2 tablespoons unsalted melted butter and 7-8 tablespoons water.

- Method of preparation:

1. Preheat the oven to $180^{\circ} \mathrm{C}$

2. In a medium bowl, mix together the flour, butter and water

3. Add powdered yeast extract, flavor and salt according to conditions

4. Turn the dough out onto a floured surface and roll out the dough as thin as possible (Figure 3)

5. Cut into pieces and place the dough pieces onto a baking sheet

6. Bake for 15 minutes not until crispy

7. Allow the crackers to cool, serve at room temperature. 


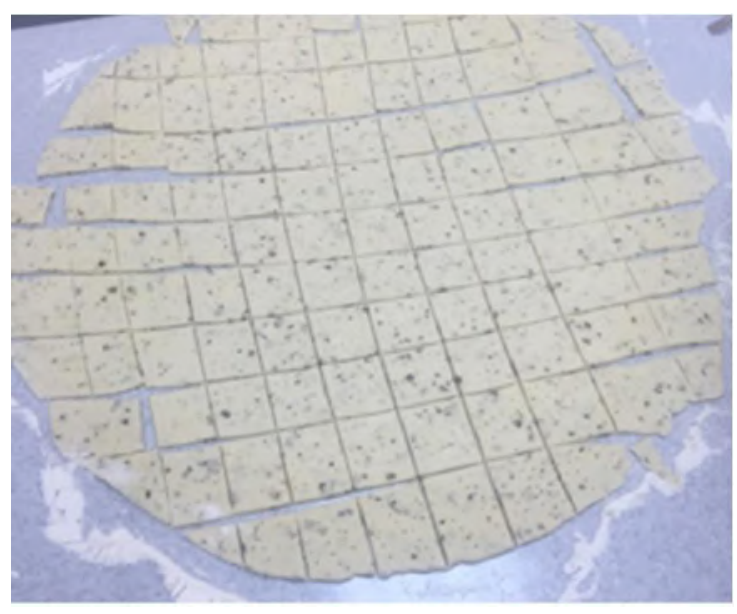

Fig.3: A sample of crackers dough cut into pieces.

\section{The Modified Baking of Crackers}

A. Determination of standard salt amount:

Prepare different dough containing different amount of salt in one cup flour. The standard salt amount was determined by five panelists aged between $25-35$, to be $7.5 \mathrm{~g}$ salt per one cup flour.

B. Determination of the flavor to be used:

Prepare cracker dough as described above. Try different flavors like olive, tomato, basil, garlic, cinnamon and ginger. We decided to use the basil as a flavor based on a sensory preference test performed for 5 panelists.

C. Modified recipe:

For the purpose of testing the salting effect of yeast extract, of different concentrations, we prepared five different dough (Table 5). The variables are the amount of salt and yeast extract added to each. All the dough included $2 \mathrm{~g}$ basil as a flavor.We substituted the amount of salt with powdered yeast extract as follows:

Table 3: The different concentrations of salt and YE used in each batch of crackers baking.

\begin{tabular}{|c|c|c|}
\hline & Salt & Yeast extract \\
\hline Control & $100 \%$ & $0 \%$ \\
\hline Sample 1 & $75 \%$ & $25 \%$ \\
\hline Sample 2 & $50 \%$ & $50 \%$ \\
\hline Sample 3 & $25 \%$ & $75 \%$ \\
\hline Sample 4 & $0 \%$ & $100 \%$ \\
\hline
\end{tabular}

Sensory Evaluation

Werecruited panellists 25 males and females, aged between 18 and 25 to conduct the sensory evaluation

Two tests were conducted:
- The first one is triangle test. Four different runs were made:

$>$ Control and sample 1

$>$ Control and sample 2

$>$ Control and sample 3

$>$ Control and sample 4

In each run, each panelist was subjected to a random set of three crackers, two different and one odd. Each panelist was asked to determine the odd sample and record his answer in a sheet.

Three coding system were used and each cracker was given a number of three digits randomly.

- The second test is the consumer acceptance test. The panelists were given one sample in each run and asked to evaluate different features of the cracker on a hedonic scale from 0 to 9 .

\section{RESULTS AND DISCUSSION}

Despite its numerous potential effects, the usage of yeast extract in the food industry still limited. The main objective of this study was to shed the light over the potential saltiness of yeast extract, which goes far beyond using it as a simple food additive for enhancing the flavor or giving Umami taste. Being mainly an ingredient used in bakery and brewery, yeast has much more potential uses to be investigated. The hypothesis that YE can partially replace salt in salty crackers has been supported by our results.

\section{Preparation of Yeast Extract}

Autolysis

When $125 \mathrm{~g}$ of dried yeast dissolved in $500 \mathrm{ml}$ distelled water, a volume of $250 \mathrm{ml}$ brown liquid extract is observed. The yeasts are continuously heated at $45^{\circ} \mathrm{C}$ and incubated for four days. According to the literature the most important condition for autolysis is the temperature (Luna-Solano et al., 2005). The optimal temperature determined by Padmakumara has been set at $45^{\circ} \mathrm{C}$. Due to its convenience, this value has been also adopted to our experimental protocols. This process is enzyme driven where the catalytic activity is affected by temperature, at which it can reach its highest amplitude (Padmakumara, 2006). The endogenous enzymes come in contact with the cell wall, starting to break down the yeasts wall, allowing them to release all their content outside. In our experiment, we adopted the protocol applied by Zareiusing500g of baker's yeast (Saccharomyces cerevisiae) to $2 \mathrm{~L}$ of distilled water.After four days, the extract was purified by centrifugation to allow the cell debris settle to the bottom. The supernatant or the extract 
was then stored in a liquid form at $-20^{\circ} \mathrm{C}$ until preparation of the yeast extract in powder form (Zarei et al., 2016).

\section{Spray Drying}

Spray drying is a commonly used protocol in food industry. In this process a solution of a desired compound is transferred into powder form using a heated air(LunaSolano et al., 2005). The water in the solution is totally evaporated; this explains the increase in saltiness of the yeast extract after being spray dried. From a starting volume of $800 \mathrm{ml}$ of liquid yeast extract we were able to collect $10 \mathrm{~g}$ of powder that had more saltiness and more intenseflavor. The advantage of the spray drying is that we were able to work with a powder form of YE, that has an intense flavor of yeast, showed more saltiness, and has a longer shelf life like all powdered products. The limiting factor of this technique could be related to the need of large extract volume to be spray dried into powder.

\section{Characterization of Yeast Extract}

\section{Salinity of yeast extract}

Saltiness in the liquid yeast extract is measured by the electro conductivity (EC) meter. The salts are made of ions, and the EC measures the flowing electrical current between two metal plates (electrodes) in a liquid sample (Semat \& Katz, 1958). The more dissolved salt in water, the stronger the current flow and the higher the EC. In our study we reported the EC of different concentrated samples of liquid yeast extract, and converted the EC to concentration of ions in $\mathrm{g} / \mathrm{L}$. When the yeast extract is concentrated from a volume of $100 \mathrm{ml}$ to $80 \mathrm{ml}$, the EC recorded a value of $12.45 \mathrm{~ms} / \mathrm{cm}$ corresponding to $7.96 \mathrm{~g} / \mathrm{L}$ of salts. The higher salt concentration was recorded when the solution is concentrated to a volume of $40 \mathrm{ml}$, where the EC recorded $17.9 \mathrm{~ms} / \mathrm{cm}$. However when the solution has been concentrated to $20 \mathrm{ml}$, the EC could not record any flow of current in the first replicate, probably because the liquid became thick and many substances are broken or dissolved under heat effect. Golnabi recorded the EC of solutions with different concentrations of $\mathrm{NaCl}$. The electrical conductivity of $0.025 \mathrm{~mol} / \mathrm{L} \mathrm{NaCl}$ is about 2.807 $\mathrm{ms} / \mathrm{cm}$. This value increases as concentration increases to reach a value of $37.242 \mathrm{~ms} / \mathrm{cm}$ for $1 \mathrm{~mol} / \mathrm{L} \mathrm{NaCl}$ (Golnabi et al., 2009). When Golnabi ran the EC in $0.2 \mathrm{~mol} / \mathrm{L} \mathrm{NaCl}$ solution, a value of $18 \mathrm{~ms} / \mathrm{cm}$ was recorded. This value is equal to the one obtained in our experiment when we concentrated our YE solution to a value of $40 \mathrm{ml}$. We can sum up that $40 \mathrm{ml}$ of our solution when contained approximately $0.2 \mathrm{~mol} / \mathrm{L} \mathrm{NaCl}$. This is good evidence that the YE contains salty particles, and it is confirmed that when we concentrate the YE we can get a certain level of saltiness. However, heating the solution for a long period may damage some of the cell content and lead to significant difference in the flavor of the powder.

\section{pH of Yeast Extract}

The $\mathrm{pH}$ has been evaluated using a $\mathrm{pH}$ meter. The $\mathrm{pH}$ is a primary indicator about the acidity of a solution. The results showed that the $\mathrm{pH}$ increases from 5.19 to 5.53 as the volume of the yeast extract decreases from $30 \mathrm{ml}$ to $6 \mathrm{ml}$. Zarei in his study found that the $\mathrm{pH}$ of the yeast extract was 6.29 (Zarei et al., 2016). This indicates that our product is slightly more acidic, so adding some sodium bicarbonate to the recipe tends to cover the acidic taste of the YE.

\section{Determination of Fat by Soxhlet}

Soxhlet extraction is one of the most commonly used methods for determination of total fat. It is associated with solvent extraction (Saunders, 1962). There are two ways to calculate the fat percentage by using the soxhlet. In the first method we used the mass of the flask were the extracted fat should be settled. The fat percentage was $3.96 \%$. This percentage was higher than the calculated percentage using the second practice $(3.4 \%)$; based on calculating the mass of the sample before and after fat extraction. Zarei tested the fat percentage of his powdered yeast extract that is produced by autolysis too, and found it to be $0.42 \%$, lower than our results. This difference can be due to the autoclaving factor, where Zarei used autoclaving and fast cooling twice while preparing the YE. This might affected the action of the yeast cells, where they did not release all their content to the media. Despite this difference, both obtained values showed that YE is low in fat product.

\section{Determination of Total Nitrogen by Kjendahl, Distillation and Titration}

The first step is a process of digestion, where the entire nitrogen bonds are broken, and the organically bound nitrogen is converted into ammonium ions $\left(\mathrm{NH}_{4}{ }^{+}\right)$. The Kjendahl tablets were used as a catalyst to speed up the digestion. The higher the temperature, the faster the digestion is, thus the temperature was set to $420^{\circ} \mathrm{C}$ (based on the manual of the machine). During digestion, foam appeared at the surface of the digest, and a clear solution indicates the completion of the digestion. After running the process for 4.5 hours, the sample is allowed to cool at room temperature.

The second step is a process of distillation. $\mathrm{NaOH}$ is used to convert ammonium ions $\left(\mathrm{NH}_{4}{ }^{+}\right)$into ammonia $\left(\mathrm{NH}_{3}\right)$. The final step is dedicated for the titration, where the sample is titrated against $\mathrm{HCl}$, in order for the amount of nitrogen to be determined(Swoboda, 1922). Using the law of equivalence, at equivalence point, the number of moles 
of nitrogen is equal to the number of moles of $\mathrm{HCl}$, the concentration of nitrogen was determined to be 0.03192 M. Comparing our results to those of Zarei, who found that the percentage of total nitrogen is $9.2 \%$, we can conclude that our YE contain lower amount of nitrogen. We multiplied our results by a factor of 6.25 to calculate the amount of protein, and the value was $0.2 \mathrm{~g} / 100 \mathrm{~g}$. This value indicated that the YE contains an accepted amount of proteins

\section{Determination of Nucleotides}

The nucleotides 5'IMP (inosine 5'-monophosphate) and 5'GMP (guanosine 5'-monophosphate) are responsible of the intensification of naturalflavor and for the enhancement of Umami taste in the yeast extract(Pereira et al., 2018). The yeast contains about 7-12\% RNA (Mutiu \& Brandl, 2005). We followed the protocol of Pereira to determine the amount of these nucleotides by spectrophotometric methods. The previous records of the nucleotidesabsorbance varies within the range of 200$300 \mathrm{~nm}$, with a peak at 250nm (Pereira et al., 2018). The measured absorbance of the nucleotides in our yeast extract has been found to be 3.00 at $250 \mathrm{~nm}$. By substituting the absorbance of the yeast extract in the equations of both curves (figure 3 and 4), we reported the value of $1.82 \mathrm{M}$ for 5 'IMP and $1.38 \mathrm{M}$ for 5'GMP in our yeast extract. These results explained the intensified Umami taste of our YE, and showed that it may contain natural flavoring content that improves the taste of the food.

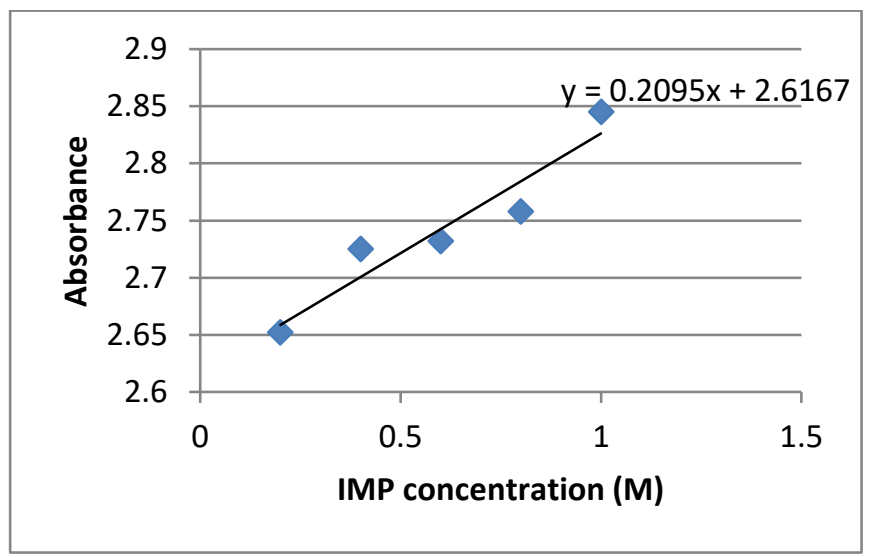

Fig.3: The standard curve determined by measuring the absorbance of different concentrations of IMP at 250nm.

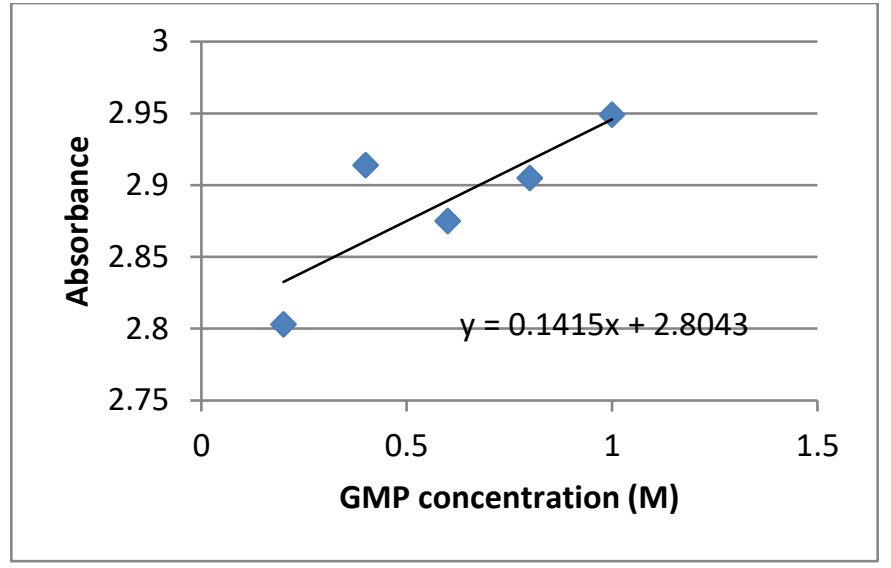

Fig.4: The standard curve determined by measuring the absorbance of different concentrations of GMP at 250nm.

\section{Determination of Glucose by Refractometer}

Refractometer is a method used to measure the Refractive Index of a particular substance. We prepared different concentrations of glucose $(0.1-1 \mathrm{~g} / \mathrm{ml})$ to draw the standard curve. The plot of the standard solution yielded a straight line with $\mathrm{R}^{2}$ of 0.9771 (figure 5). This regressed line was used to determine the amount of sugar in our yeast extract, found to be is $1.4 \mathrm{~g} / \mathrm{ml}$. This finding promotes the use of $\mathrm{YE}$ as a healthy product that is very low in glucose level.

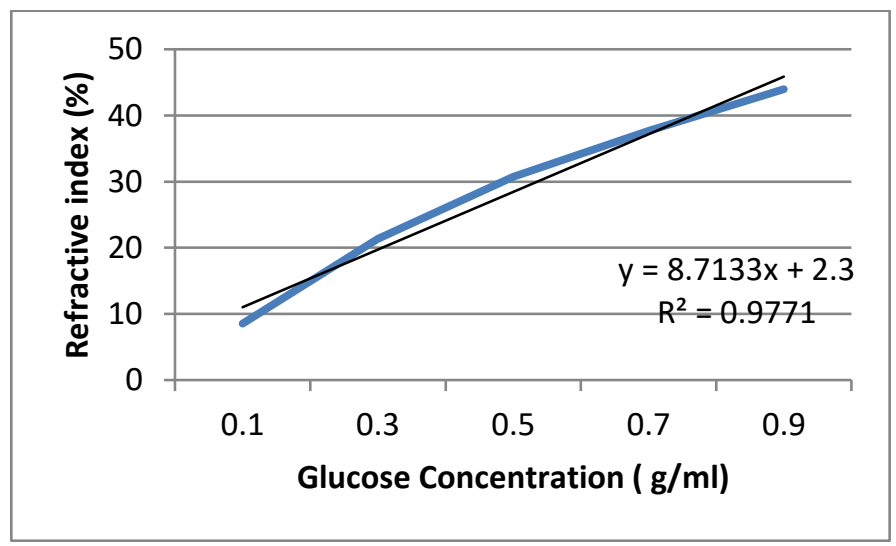

Fig.5: The standard curve was determined by measuring the refractive index of different glucose concentrations.

\section{Determination of Glutamic Acid}

Glutamic acid could be found naturally in different kinds of food like meat, poultry, cheese, milk and tomatoes (Kulkarni et al., 2005). Early it has reported that glutamic acid can react with the acetylacetone- formaldehyde reagent to give a yellow color(Shah, 1992). In his study, Shah and his colleagues determined the optimal conditions for that reaction and for obtaining the more intensified yellow color. A maximal value of glutamic acid absorbance was obtained at $415 \mathrm{~nm}$ using $3 \mathrm{ml}$ of the 
reagent, heated at $100^{\circ} \mathrm{C}$ in a boiling water bath for 20 minutes.

In our study we followed the protocol described by Shah, and we used his data to define the reaction parameters. At the end of the reaction, the absorbance of the dried yeast extract has been found to be 1.2383 at $415 \mathrm{~nm}$. Based on the standard curve using acetylacetone- formaldehyde, the plot followed a regression line with $\mathrm{R}^{2}=0.9696$ (figure 6). The concentration of glutamic acid in our dried yeast extract was $0.689 \mathrm{~g} / \mathrm{ml}$. Therefore, our YE not only can be used to give Umami flavor, but also might be added to almost all foods as a flavor enhancer. This shows that our product could be a potential substitute of MSG in food industry.

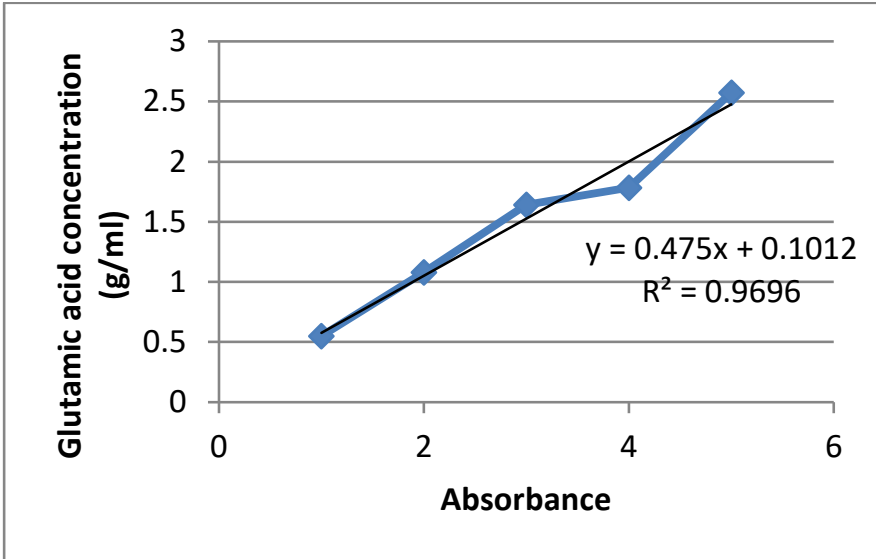

Fig.6: The standard curve determined by measuring the absorbance of different concentrations of Glutamic acid at $415 \mathrm{~nm}$.

\section{Sensory Analysis}

\section{Triangle Test}

The first triangle test was conducted between the control and sample 1 ( $25 \%$ YE, $75 \%$ salt). Only seven panelists out of 25 were not able to identify the different sample, and the results showed that there is a significant difference between the control and sample 1. These results indicated that crackers samples containing a ratio of 1:3 (YE: Salt) were easily differentiated by panelists who were able to feel the difference in flavor.

The second run was conducted to compare the control with sample 2 (50\% YE, 50\% salt). Out of 25 panelists, 19 ones gave a wrong answer and could not identify the odd sample between the set and the difference was not significant. When the amount of YE was increased and substituted $50 \%$ of salt, its saltiness increased and the flavor was more intensified. Using this protocol, we could reduce the amount of salt in crackers to the half, and substitute it with a natural product, the YE, maintaining the same flavor of the product.
The third run was conducted between the control and sample 3 (3:1) (YE: Salt). The number of correct answers was 23 . Only 2 panelists were not able to identify the different sample (control). The difference was significant between the control and this sample. So by substituting $75 \%$ of the initial salt amount by YE, the salty flavor was affected and the difference was apparent. The flavor of YE masked its saltiness, that's why the panelists were able to spot the odd cracker. This difference was on the level of flavor where the flavor of YE was apparent, not only on the level of saltiness.

The final run of the test was conducted between the control and the sample 4 (100\% YE). Only one person was not able to identify the odd sample, while 24 panelists could recognize the different cracker. The difference was highly significant, and the two samples showed a huge difference between them. When salt is $100 \%$ substituted by YE the flavor and saltiness were totally different compared to the control.

\section{Consumers' acceptance test}

This test was conducted to evaluate how much consumers will like the edited crackers at the level of the flavor, saltiness and overall acceptability. The first test was held using sample 2 that is made up of 50\% salt and 50\% YE. The panelists were asked to score each trait on a hedonic scale from 1 (dislike extremely) to 9 (like extremely). At the level of saltiness, sample 3 scored 6.4 over 9 , which is nearer to slightly like (on the scale). At the level of saltiness, it scored 6.48 over 9 . The overall acceptability scored 6.28 over 9 . These results are acceptable for a product for which, the salt content is reduced to the half.

The second test was conducted using sample 3 made up of $25 \%$ salt and $75 \%$ YE. The same hedonic scale was used as a reference. At the level of the saltiness, this sample scored 5.36 over 9 (neither like, nor dislike). Its saltiness scored 4.96 over 9 , which are lower than sample 2. The overall acceptability was 5.48 over 9 .

The numbers above are good evidence that reducing salt by half and replacing the reduced amount by YE gives a successful healthy salty cracker. The ratio of 2:2 (YE:Salt) gave a promising results that replacing salt by YE could be helpful in producing healthy products, while when the ratio was 3:1 (YE:Salt) the results showed that there is a significant difference between samples and thus the edited crackers were not helpful.

\section{CONCLUSION AND FUTURE STUDIES}

Yeast extract proved its efficacy in replacing salt. Our results confirmed that consumers liked the crackers made up of $50 \%$ salt and $50 \%$ YE, and they showed no 
significant difference with the control made up of $100 \%$ salt. Moreover, the crackers where $25 \%$ of the salt was replaced by YE showed a significant difference with the control. Therefore, YE can replace salt at a specific level and amount. The physiochemical properties of YE are interesting with very low amount of fat, glucose and calories; whereas the count of total nitrogen, nucleotides and glutamic acid were acceptable. The $\mathrm{pH}$ was acidic (5), and the liquid YE contained approximately $9 \mathrm{~g} / \mathrm{L}$ of salt. The presence of nucleotides and glutamic acid in the YE gave it the flavor enhancing property. In addition to that, the dried powdered YE gave a more salty flavor than the liquid YE; this can be explained by the higher concentration in active ingredients and a total evaporation of the water. Future studies should focus on using YE to replace salt not only for its salty taste, but also to intensify and enhance flavors of food when added. Also, the effect of $\mathrm{YE}$ in causing allergy to some people may have a great interest.

\section{REFERENCES}

[1] Golnabi, H., M.r, M., M, B., \& M, S. (2009). InvestigationofElectricalConductivityof Different Water Liquidsand Electrolytes Solutions. 3(2), 24-28.

[2] Henney, J. E. (2010). Strategies to Reduce Sodium Intake in the United States (J. E. Henney, C. L. Taylor, \& C. S. Boon, Eds.). National Academies Press (US). http://www.ncbi.nlm.nih.gov/books/NBK50956/

[3] Kulkarni, C., Kulkarni, K. S., \& Hamsa, B. R. (2005). LGlutamic acid and glutamine: Exciting molecules of clinical interest. Indian Journal of Pharmacology, 37(3), 148. https://doi.org/10.4103/0253-7613.16210

[4] Laulloo, S., \& Cadet, F. (2003). Quantitative determination of sugar in fruits by different methods. ResearchGate. https://www.researchgate.net/publication/258844672_Quan titative_determination_of_sugar_in_fruits_by_different_me thods

[5] Lehnhardt, A., \& Kemper, M. J. (2011). Pathogenesis, diagnosis and management of hyperkalemia. Pediatric Nephrology (Berlin, Germany), 26(3), 377-384. https://doi.org/10.1007/s00467-010-1699-3

[6] Liem, D. G., Miremadi, F., \& Keast, R. S. J. (2011). Reducing sodium in foods: The effect on flavor. Nutrients, 3(6), 694-711. https://doi.org/10.3390/nu3060694

[7] Luna-Solano, G., Salgado-Cervantes, M. A., RodríguezJimenes, G. C., \& García-Alvarado, M. A. (2005). Optimization of brewer's yeast spray drying process. Journal of Food Engineering, 68(1), 9-18. https://doi.org/10.1016/j.jfoodeng.2004.05.019

[8] Mihalos. (2014). Mihalos-Cracker-Processing-BiscuitTechnology-2014-TC.pdf | Dough | Mondelez International. Scribd. https://www.scribd.com/document/367663550/MihalosCracker-Processing-Biscuit-Technology-2014-TC-pdf
[9] Mihiranie, S., Jayasundera, M., \& Perera, N. (2017). Developmentof Snack Crackers Incorporatedwith Defeated Coconut Flour. https://doi.org/10.15414/jmbfs.2017.7.2.153-159

[10] Mutiu, A. I., \& Brandl, C. J. (2005). RNA Isolation from Yeast Using Silica Matrices. Journal of Biomolecular Techniques : JBT, 16(4), 316-317.

[11] Padmakumara, U. (2006). Manufacture Of Edible Yeast Extract From Brewery Waste Yeast. University of Sri Jayewardenepura.

[12] Pereira, R. A. F., Ugucione, C., \& Pereira-Filho, E. R. (2018). Determination of inosine 5'-monophosphate (IMP) and guanosine 5'-monophosphate (GMP) in yeast extracts using UV spectroscopy and chemometrics. Brazilian Journal of Food Technology, 21(0). https://doi.org/10.1590/1981-6723.12717

[13] Saunders, F. (1962). Studies On The Fatty Acid Composition Of Yeast..

[14] Semat, H., \& Katz, R. (1958). Physics, Chapter 28: Electrical Conduction in Liquids and Solids. 16.

[15] Shah, S. (1992). Spectrophotometric Determination of Amino Acids and Their Dosage Forms. Indian Journal of Pharmaceutical Sciences.

[16] Sunil, P., Goel, A., \& Rizvi, Y. S. (2013). Salt substitutes: Are they safe? Indian Journal of Nephrology, 23(3), 238. https://doi.org/10.4103/0971-4065.111871

[17] Swoboda, F. K. (1922). Nitrogen Nutrition of Yeast. Journal of Biological Chemistry, 52(1), 91-109.

[18] Tangüler, H., \& Erten, H. (2009). The Effect of Different Temperatures on Autolysis of Baker's Yeast for the Production of Yeast Extract. Turkish Journalof Agricultureand Forestry, 33(2), 149-154.

[19] Taylor, C., Doyle, M., \& Webb, D. (2018). "The safety of sodium reduction in the food supply: A cross-discipline balancing act"-Workshop proceedings. Critical Reviews in Food Science and Nutrition, 58(10), 1650-1659. https://doi.org/10.1080/10408398.2016.1276431

[20] Trayes, K. P., Studdiford, J. S., Pickle, S., \& Tully, A. S. (2013). Edema: Diagnosis and Management. American Family Physician, 88(2), 102-110.

[21] Zarei, O., Dastmalchi, S., \& Hamzeh-Mivehroud, M. (2016). A Simple and Rapid Protocol for Producing Yeast Extract from Saccharomyces cerevisiae Suitable for Preparing Bacterial Culture Media. Iranian Journal of Pharmaceutical Research : IJPR, 15(4), 907-913. 